\title{
EL ACUERDO DE PARÍS. PREDOMINIO DEL SOFT LAW EN EL RÉGIMEN CLIMÁTICO*
}

\author{
THE PARIS AGREEMENT. THE PREDOMINANCE OF SOFT LAW \\ IN THE CLIMATE REGIME
}

\section{César NAva Escudero**}

RESUMEN: Es práctica común entre los Estados, adoptar dentro del régimen internacional de protección al ambiente, al que pertenece el régimen climático, acuerdos vinculantes con un alto contenido de normas que carecen de obligatoriedad; esto es, normas de soft law. El Acuerdo de París de 2015 es precisamente un ejemplo de lo anterior. A través de un breve análisis del contenido soft en los dos antecedentes convencionales más importantes (es decir, la Convención Marco de las Naciones Unidas sobre el Cambio Climático de 1992 y el Protocolo de Kioto a dicha Convención de 1997), este artículo tiene por objeto examinar algunos de los preceptos más relevantes del Acuerdo de París, para corroborar la reincidencia de los Estados en el uso de normas no vinculantes para este tipo de tratados. Esto evidencia que dicho acuerdo no es, jurídicamente hablando, un instrumento excepcional, sino tan sólo la manifestación reiterada de la comunidad internacional para enfrentar de esta manera el cambio climático.
ABSTRACT: For some time now international environmental treaties, especially those related to the climate regime, have incorporated soft law texts i.e. norms in a non-binding written form. The 2015 Paris Agreement constitutes an example of the latter. This article seeks to examine, through a brief analysis of the soft contents of the 1992 United Nations Framework Convention on Climate Change and the 1997 Kyoto Protocol, some of the most relevant norms of the Paris Agreement in order to provide evidence that on securing agreement on climate issues States resort to the use of non-binding norms. This common practice proves that, at least in legal terms, the Paris Agreement is not an exceptional treaty as conceitedly described.

* Artículo recibido el 15 de febrero de 2016 y aceptado para su publicación el 6 de junio de 2016.

** Doctor $(\mathrm{PhD})$ por la London School of Economics and Political Science, Inglaterra; investigador titular en el Instituto de Investigaciones Jurídicas de la UNAM, y pertenece al SNI, nivel II. 
Esta revista forma parte del acervo de la Biblioteca Jurídica Virtual del Instituto de Investigaciones Jurídicas de la UNAM

Palabras clave: Acuerdo de París, soft law, régimen climático, derecho internacional ambiental.
Keywords: Paris Agreement, soft law, climate regime, international environmental law.

SUMARIO: I. Introducción. II. Sobre los tratados y la naturaleza de sus normas. III. El soft law en el derecho internacional ambiental. IV. El Acuerdo de París: reincidencia del soft law en el régimen climático. V. Reflexión final. VI. Bibliografia.

\section{INTRODUCCIÓN}

Entre aplausos, ovaciones y algunos llantos de alegría, líderes y políticos del mundo y representantes de 195 países festejaron el 12 diciembre de 2015 la aprobación del tan esperado acuerdo jurídico sobre el clima: ${ }^{1}$ el Acuerdo de París (en adelante, AP). El escenario fue la 21 a. Conferencia de las Partes (COP-21) de la Convención Marco de las Naciones Unidas sobre Cambio Climático (en adelante, CMNUCG), que fungió también como la 11 a. Reunión de las Partes (CMP-11) en el Protocolo de Kioto a la CMNUCG (en adelante, PK), celebrada en París, Francia. La difusión en medios de comunicación masiva del éxito alcanzado no logró opacar, sin embargo, las voces disidentes de científicos, académicos o activistas que no otorgaron su beneplácito. Frente a las frases de "el mundo ha escrito una nueva página de su historia" (François Hollande), "es el marco sostenible que el mundo necesita para resolver la crisis climática" (Barack Obama), o "es un triunfo monumental para los pueblos y para nuestro planeta" (Ban Ki-Moon), surgieron las de "estamos ante un fraude y una farsa... no hay acciones sólo promesas" (James Hansen), la cumbre fracasó "antes de empezar" (Naomi Klein), o "nada va a ocurrir sustancialmente hasta 2020" (Ilan Kelman). ${ }^{2}$

Desde la perspectiva estrictamente jurídica, tanta algarabía tiene mucho de su fundamento en el hecho de que se haya aprobado un instru-

1 Enfatizamos el "tan esperado" porque desde el 2009 (COP-15 en Copenhague) y luego el 2010 (COP-16 en Cancún) se tuvo tal expectativa. Los documentos aprobados en ellas no alcanzaron el estatus vinculante.

2 Las frases atribuidas a F. Hollande (presidente de Francia), B. Obama (presidente de Estados Unidos), Ban Ki-Moon (secretario general de la ONU), J. Hansen (profesor en la Universidad de Columbia), N. Klein (activista canadiense) e I. Kelman (investigador en la University College London) están en wrere.elmundo.es y wrere.excelsior.com.mx del 13 de diciembre, y wrwr.bb.com del 14 del mismo mes, todas de 2015. 
mento internacional de naturaleza vinculante, con el objeto de mejorar la aplicación de la CMNUCC, y de reforzar con ello la respuesta global al cambio climático. A priori, esto significa que las partes contratantes habrían manifestado su voluntad para crear preceptos de carácter obligatorio - normas de hard law - en el texto de un instrumento considerado tratado en todos los sentidos. Sin embargo, tanto optimismo oculta, o ignora, que es práctica común entre los Estados en el régimen internacional de protección al ambiente (al cual pertenece el régimen climático) el adoptar acuerdos vinculantes con un alto contenido de normas que, en realidad, carecen de obligatoriedad — normas de soft law-.

Este artículo tiene por objeto explicar, con base en un examen sobre la naturaleza jurídica de las normas en los tratados, la existencia y predominio del soft law en el derecho internacional ambiental y en lo particular en el régimen climático. A través de un breve análisis del contenido soft en los dos antecedentes convencionales más importantes en la materia (es decir, la CMNUCG de 1992 y el PK de 1997) se estudian algunos de los preceptos más relevantes del AP de 2015 para corroborar la reincidencia de los Estados en el uso de normas no vinculantes para este tipo de tratados. Esto evidencia que dicho acuerdo no es, jurídicamente hablando, un instrumento excepcional o histórico, sino tan sólo la manifestación reiterada de la comunidad internacional para enfrentar de esta manera el cambio climático. Por lo tanto, en el contexto jurídico, las expresiones de triunfalismo se antojan exageradas y engañosas.

\section{SOBRE LOS TRATADOS Y LA NATURALEZA DE SUS NORMAS}

Es aceptado entre los especialistas de derecho internacional, que una de las características o elementos esenciales de los tratados es la de producir efectos jurídicos, es decir, la de crear obligaciones entre los Estados que los celebran.

El tratado internacional otorga derechos e impone obligaciones a las partes contratantes, preferentemente. Es una regla de conducta obligatoria para los Estados que los suscriben y ratifican. La teoría de la fuerza obligatoria de los pactos internacionales ha sido muy amplia y se ha orientado hacia los más variados criterios... pero la esencia de ellas es la afirmación del ca- 
rácter obligatorio de los tratados, cualquiera que sea el fundamento que las informa. ${ }^{3}$

Descansan los tratados en tres principios: 1) todo tratado al entrar en vigor obliga a las partes y debe cumplirse de buena fe (pacta sunt servanda); 2) un tratado produce efectos únicamente entre las partes que lo celebran (res inter alios acta): en principio, no crean obligaciones para un tercer Estado sin su consentimiento, y 3) el consentimiento es la base de las obligaciones convencionales: del consentimiento deviene la obligación (ex consensu advenit vinculum $).{ }^{4}$ Se entiende, por tanto, que los tratados son instrumentos internacionales, o como los llama la Convención de Viena sobre el Derecho de los Tratados de 1969 (en adelante, CV de 1969), acuerdos internacionales, ${ }^{5}$ que son obligatorios, es decir, vinculantes. Son usualmente denominados por la doctrina como instrumentos de derecho duro o fuerte, en inglés, hard law.

Con el objetivo de brindar una visión general de términos clave que se emplean en su Colección de Tratados, la Organización de las Naciones Unidas ha señalado que el vocablo "tratado" se utiliza ya sea de forma particular para designar un instrumento con características definidas, o bien de forma genérica para referirse a cualquier instrumento vinculante en el derecho internacional que haya sido concluido entre entidades internacionales. ${ }^{6}$ En este último supuesto, para que se considere tratado, un instrumento internacional debe cumplir, entre otros, con el criterio de que

3 Sepúlveda, César, Derecho internacional, 20a. ed., México, Porrúa, 2000, p. 139. En esta obra, al principio, se hace alusión a las doctrinas que abordan la teoría de la fuerza obligatoria de los tratados.

4 Méndez-Silva, Ricardo, "Tratados internacionales", Diccionario jurídico mexicano, 8a. ed., México, Porrúa-UNAM, Instituto de Investigaciones Jurídicas, 1995, pp. 3150 y 3151. En este mismo sentido, véase Seara Vázquez, Modesto, Derecho internacional público, 24a. ed., México, Porrúa, 2012, pp. 71 y 72.

5 La CV de 1969 (en vigor desde el 27 de enero de 1980) establece en su artículo 2o. que tratado es "un acuerdo internacional celebrado por escrito entre Estados y regido por el derecho internacional, ya conste en un instrumento único o en dos o más instrumentos conexos y cualquiera que sea su denominación particular".

6 Sigue consecuentemente lo establecido al respecto tanto por la GV de 1969 como por su similar de 1986, es decir, la Convención de Viena sobre el Derecho de los Tratados entre Estados y Organizaciones Internacionales o entre Organizaciones Internacionales de 1986, que aún no entra en vigor. 
Esta revista forma parte del acervo de la Biblioteca Jurídica Virtual del Instituto de Investigaciones Jurídicas de la UNAM

sea vinculante, lo que ocurre cuando las partes contratantes se han comprometido a crear derechos y obligaciones jurídicas. ${ }^{7}$

En realidad, no es relevante para el derecho internacional el nombre con el que se bautice a un tratado. ${ }^{8}$ En efecto, ya desde los trabajos realizados por la Comisión de Derecho Internacional hace algunas décadas, y ahora por norma convencional, un tratado puede adoptar cualquier denominación: acuerdo, convenio, convención, protocolo, pacto, carta, declaración, memorandum de entendimiento, modus vivendi, canje de notas, estatuto, acta o, incluso, tratado, entre otras. ${ }^{9}$ Lo que es relevante, como ya se dijo, es que para que un instrumento internacional se considere como un tratado deberá tener per se fuerza jurídica vinculante; ésa es la esencia del tratado. De aquí que la expresión "tratado vinculante" o "tratado de derecho duro" sea tautológica: es una acumulación reiterativa de términos.

Siguiendo lo anterior, dos instrumentos pueden tener un mismo sustantivo, pero uno ser tratado y otro no. Por ejemplo, la Carta de la Organización de las Naciones Unidas es (y se considera) un tratado, ${ }^{10}$ pero no así la Carta Mundial de la Naturaleza. ${ }^{11}$ Ambos instrumentos internacionales se llaman carta, pero uno es obligatorio y el otro no. Para estos casos, como en otros, lo que es determinante para el estatus del documento no es el título que adquiera, sino la intención de las partes para crear relaciones jurídicamente vinculantes. ${ }^{12}$ Dicho de otra manera, el nombre del instrumento no necesariamente determina su naturaleza jurídica.

7 Los otros criterios son que el instrumento debe celebrarse por Estados u organizaciones internacionales con poder de establecer tratados, debe regirse por el derecho internacional, y debe estar por escrito. Véase United Nations Organization, "Definitions of Key Terms Used in the UN Treaty Collection", United Nations, Treaty Collection, disponible en: https://treaties.un.org/Pages/overview.aspx:path=overview/definition/page1_en.xml.

8 A veces, el término "tratado" designa instrumentos sobre materias de la mayor importancia, pero se reconoce que "si bien existen ciertos usos, no puede hablarse de una práctica uniforme respecto a la manera de designar los tratados...”. Para más detalles, véase Palacios Treviño, Jorge, Tratados. Legislación y práctica en México, 4a. ed., México, Secretaría de Relaciones Exteriores-Universidad Iberoamericana, 2007, pp. 36-43.

9 Estos trabajos sirvieron de base para la elaboración de la CV de 1969. Para mayor referencia, véase Brownlie, Ian, Principles of Public International Law, 7a. ed., Oxford, Oxford University Press, 2008, pp. 607 y 608.

10 Se aprobó el 26 de junio de 1945 y entró en vigor el 24 de octubre del mismo año.

11 Aprobada el 28 de octubre de 1982 por Resolución 37/7 de la Asamblea General de la ONU.

12 Shaw, Malcolm N., International Law, 7a. ed., Cambridge, Cambridge University Press, 2014, p. 84. 
Entonces, es claro que el carácter obligatorio o hard de los tratados se manifiesta a través de preceptos de naturaleza vinculante, que son los que dotan de contenido al instrumento y que resultan del consentimiento de los Estados. La práctica internacional y la doctrina suelen referirse a ellos como normas, aunque también como compromisos, mandatos, reglas, entre otros. Este tipo de disposiciones se adjetivan de jurídicas, vinculantes, legales, obligatorias o, incluso, jurídicamente vinculantes, para diferenciarlas de aquellas que no lo son. Así, se dice que las normas contenidas en un tratado son, en principio, normas jurídicas, normas de derecho duro o fuerte, en inglés, normas hard law.

Es fundamental precisar que las obligaciones jurídicas en el derecho internacional no tienen el mismo significado y alcances que en el derecho interno, particularmente por lo que se refiere a las sanciones, a los mecanismos coactivos para el cumplimiento de las normas y, desde luego, a la naturaleza misma de las obligaciones. ${ }^{13}$ Pero no por ello la normatividad internacional carece de obligatoriedad.

En efecto, hay quienes sostienen que el fundamento de la obligatoriedad de una norma internacional es la coercibilidad, ${ }^{14}$ típica en cualquier norma de derecho: "la norma jurídica es coercible, es decir, ante su incumplimiento se actualiza la consecuencia jurídica que es la sanción. El Estado está obligado a cumplir con la norma jurídica internacional, sabedor de que si la destaca (sic) se hace acreedor a una sanción internacional". ${ }^{15}$ De forma similar, se ha señalado que el carácter jurídico del derecho internacional es determinado por la sanción, particularmente "la responsabilidad internacional que la violación del D. I. origina”, y de aquí que se pueda hablar de la existencia de coacción ${ }^{16}$ en el derecho interna-

13 Un buen análisis sobre esta cuestión se encuentra en Vallarta Marrón, José Luis, Derecho internacional público, 2a. ed., México, Porrúa, 2014, pp. 1-10, y también en López-Bassols, Hermilo, Los nuevos desarrollos del derecho internacional público, 3a. ed., México, Porrúa, 2008, pp. 31 y 32 .

14 Coerción es "el empleo necesario de la fuerza"; en cambio, coercibilidad es el "uso forzoso de la fuerza". Villoro Toranzo, Miguel, Introducción al estudio del derecho, 21 a. ed., México, Porrúa, 2012, p. 452.

15 Arellano García, Carlos, Primer curso de derecho internacional público, 7a. ed., México, Porrúa, 2013, p. 131.

16 Tradicionalmente se ha dicho que todo ordenamiento jurídico, o derecho, debe ser coactivo. Una sinonimia de coacción es la de empleo de la fuerza, y se considera como "un medio de que dispone un órgano de autoridad para hacer cumplir una norma sancionada". Villoro Toranzo, Miguel, op. cit., p. 452. 
cional. ${ }^{17} \mathrm{Y}$ es que no podría hablarse de responsabilidad de los Estados si no se aceptara la existencia misma de una posible violación del derecho internacional, la cual se entiende "por referencia a las obligaciones positivas y negativas del Derecho internacional", es decir, por la existencia de una acción o una omisión. ${ }^{18}$

Todavía más allá, hay juristas que proponen la tesis de que el carácter jurídico-obligatorio del derecho internacional se entiende aún suprimiendo la sanción como es concebida tradicionalmente en el derecho interno. Aquí, en todo caso, lo que importa es determinar si existen derechos y obligaciones entre los Estados y de que éstos acepten tal situación..$^{19}$ Aunque en principio la idea de que un tratado produzca efectos jurídicos supone que sus normas serán "exigibles en derecho con todas la consecuencias que ello implica en caso de incumplimiento", ${ }^{20}$ no necesariamente deben existir sanciones para que se le considere hard law. En esta línea de pensamiento, se argumentaría que las sanciones no son un elemento constitutivo del derecho, ciertamente no lo serían como criterio para distinguir una norma vinculante de una norma que no lo es. ${ }^{21} \mathrm{El}$ sentirse obligado por una norma cuyo cumplimiento no es opcional sino algo esperado, o mejor aún, requerido, juega un papel muy importante para determinar la naturaleza hard de una norma. ${ }^{22}$

Ahora bien, aunque el contenido normativo de los tratados sea en principio de naturaleza vinculante, estos instrumentos pueden contener disposiciones que no tengan tal naturaleza; es decir, enunciados que carezcan de obligatoriedad, de conformidad con lo señalado en párrafos anteriores. La existencia, terminología y significado de estos preceptos en

17 Diez de Velasco, Manuel, Instituciones de derecho internacional público, 16a. ed., Madrid, Tecnos, 2007, p. 74. Este autor observa que el carácter jurídico del derecho internacional está asociado a la aplicación del mismo, las consecuencias de su violación y los mecanismos para garantizar su aplicación.

18 Para mayor detalle, véase Seara Vázquez, Modesto, op. cit., pp. 373 y ss.

19 Vallarta Marrón, José Luis, op. cit., pp. 2-4.

20 Palacios Treviño, Jorge, op. cit., p. 34.

21 Peters, Anne y Pagotto, Isabella, "Soft Law as a New Mode of Governance: A Legal Perspective", NEWGOV: New Modes of Governance, 28 de febrero de 2006, p. 11, disponible en: http://dx.doi.org/10.2139/ssm.1668531.

22 Para ciertos autores, esto podría ser, en última instancia, lo que en realidad hace hard a una norma. Véase lo que al respecto ha señalado Bodansky, Daniel, The Art and Craft of International Environmental Law, Cambridge-Londres, Harvard University Press, 2010, p. 101. 
tratados ha sido discutida desde hace algunas décadas. ${ }^{23}$ Su importancia actual es inobjetable, y se les conoce comúnmente como normas de derecho suave, blando o flexible, en inglés, soft law. En ocasiones también se hace referencia a ellas como compromisos u "obligaciones".

Sin embargo, y aunque la doctrina admita su existencia, no hay consenso entre los especialistas sobre cómo adjetivar a este tipo de normas ni tampoco sobre cómo determinar si tienen o no cierto grado o nivel de obligatoriedad. Hay quienes optarían por llamarles normas, compromisos $\mathrm{u}$ obligaciones no jurídicas, no legales, no vinculantes o no jurídicamente vinculantes, y quienes preferirían referirse a ellas (conjunta o separadamente) como declarativas, políticas, programáticas, exhortativas, aspiracionales, no autoejecutables, vagas e imprecisas, menos vinculantes, o normas que generan obligaciones minimas, obligaciones de comportamiento, obligaciones generales (es decir, normas de carácter general). Ante este desacuerdo doctrinal se han creado expresiones genéricas, tales como las de legal soft law o soft law material. De aquí la existencia de un derecho suave legal o de normas jurídicas de derecho suave en los tratados.

Hay que precisar que la existencia de normas de soft law en acuerdos internacionales de hard law forma parte de un proceso mucho más amplio de creación normativa no vinculante en el derecho internacional, que estaría abarcando, por ejemplo, resoluciones no obligatorias de las organizaciones internacionales, acuerdos interestatales no normativos o no vinculantes, el derecho suave de actores no estatales, y el ya aludido soft law material..$^{24}$

Es probable que la emergencia del soft law, en general, haya comenzado a percibirse y discutirse por la doctrina (de hecho, su aparición es de tipo doctrinal y no convencional) hacia comienzos de la década de los setenta del siglo pasado, ${ }^{25}$ pero con el transcurso del tiempo se ha conver-

23 Destacan como primeros escritos sobre esto, particularmente, el de Baxter, R. R., "International Law in «Her Infinite Variety»", The International and Comparative Law Quarterly, vol. 29, núm. 4, 1980, pp. 552 y ss., y el de Chinkin, C. M., "The Challenge of Soft Law: Development and Change in International Law", The International and Comparative Law Quarterly, vol. 38, núm. 4, 1989, pp. 851 y ss.

24 Estos cuatro rubros se proponen en Toro Huerta, Mauricio Iván del, "El fenómeno del soft law y las nuevas perspectivas del derecho internacional", Anuario Mexicano de Derecho Internacional, vol. VI, 2006, p. 536.

25 Así lo han señalado Dupuy, Pierre-Marie, "Soft Law and the International Law of the Environment", Michigan Fournal of International Law, vol. 12, núm. 420, 1991, p. 420, y 
Esta revista forma parte del acervo de la Biblioteca Jurídica Virtual del Instituto de Investigaciones Jurídicas de la UNAM

tido en un tema de referencia indispensable entre los expertos del derecho internacional. ${ }^{26}$ Hoy es fácil encontrarlo mencionado en una gran cantidad de textos sobre la materia. ${ }^{27}$

Un tema central en la discusión sobre el derecho suave (que comprende el de normas soft en tratados) es la terminología y el tipo de preceptos que designa. La cuestión a resolver es, por un lado, si con esta expresión estamos frente a normas que no son de derecho, es decir, normas que no son jurídicas, y por lo tanto el uso de la palabra "derecho" o law sería incorrecto, confuso, paradójico o contradictorio, porque en la concepción tradicional la norma de derecho es vinculante: si no es vinculante, entonces no es jurídica. O si, por el contrario, el derecho debe entenderse como un conjunto de diversos tipos de normas jurídicas, las cuales se encuentran en "niveles" o "grados" distintos, donde algunas son más duras que otras, o a la inversa, algunas menos vinculantes que otras.

Para esta discusión, la doctrina se ha basado fundamentalmente en dos planteamientos. El primero de ellos, conocido como visión o modelo dicotómico (en inglés, binary view), se deriva de la teoría tradicional de las fuentes del derecho internacional, ${ }^{28} \mathrm{y}$ consiste, grosso modo, en que la esencia del derecho es su normatividad vinculante, no está sujeta a gradación, y, por lo tanto, una norma de derecho o es jurídica o no lo es. La expresión soft law debiera, en este sentido, evitarse, puesto que conduce a una con-

Klabbers, Jan, "Reflections on Soft International Law in a Privatized World", Lakimies, vol. 104, 2006.

26 Algunas referencias se citan en Shelton, Dinah, "Comments on the Normative Challenge of Environmental «Soft Law»”, en Kerbrat, Yann y Maljean-Dubois, Sandrine (eds.), The Transformation of International Environmental Law, Oxford-París, A. Pedone \& Hart, 2011, p. 61, y Toro Huerta, Mauricio Iván del, op. cit., pp. 516 y ss.

27 Por ejemplo, Casese, Antonio, International Law, 2a. ed., Oxford, Oxford University Press, 2005, pp. 196 y 197; Dixon, Martin et al., Cases \& Materials on International Law, 5a. ed., Oxford, Oxford University Press, 2011, pp. 48-52; Herdegen, Matthias, Derecho internacional público, trad. de Marcela Anzola, México, Fundación Konrad Adenauer-UNAM, Instituto de Investigaciones Jurídicas, 2005, pp. 163 y 164; López-Bassols, Hermilo, op. cit., pp. 58 y 59; Shaw, Malcolm N., op. cit., pp. 83 y 84.

28 En efecto, se ha sostenido que tal "modelo de aproximación al fenómeno desde la contraposición formal soft law/hard law busca identificar como derecho (hard) solamente aquellas normas que hayan sido producidas mediante las denominadas «fuentes» tradicionales del derecho internacional, en particular, mediante los tratados y la costumbre, dejando fuera del ámbito de lo jurídico otras manifestaciones de voluntad de los sujetos de derecho internacional". Toro Huerta, Mauricio Iván del, op. cit., p. 524, y para la cita textual, p. 528. 
tradicción lógica. El segundo de los planteamientos, denominado visión o modelo de gradación normativa, normatividad graduada y variable, o simplemente continua (en inglés, graduated and diverse normativity o continuum view), radica, en términos generales, en que el derecho puede tener una variedad de impactos y efectos legales, directos e indirectos, algunos de ellos más rigurosos que otros. Aquí se reconoce que el derecho (una norma jurídica) puede ser más fuerte o más suave. Existe un continuo entre lo soft y lo hard, que está en constante evolución: no hay polarización. Representa la realidad y la práctica internacionales en su diversidad, y así, evita que la normatividad sea encapsulada en categorías tan simplistas como lo jurídico y lo no jurídico, lo legal y lo no legal. El binomio hard/soft sería inadecuado e insuficiente para describir y evaluar la norma internacional. ${ }^{29}$

Estos planteamientos han generado tipologías diversas, que intentan explicar su naturaleza y terminología. En un primer ejemplo ${ }^{30}$ se distingue entre normas de soft law y de hard law, por un lado, y entre normas de soft law y normas no legales (políticas, morales, éticas, etcétera), ${ }^{31}$ por el otro. Mientras que en este último supuesto el soft law debe y puede distinguirse de los preceptos meramente políticos (según la cercanía de lo soft con lo que se llegue a definir como derecho), en aquél no existe entre el soft law y el hard law una línea clara de diferenciación: los textos legales pueden ser más fuertes o más suaves. De cualquier modo, en ninguno de estos dos supuestos se podrían hacer delimitaciones precisas.

En otro ejemplo ${ }^{32}$ se distingue entre el legal soft law y el non-legal soft law. La primera se refiere a preceptos (reglas de conducta) en tratados suscritos

29 Para un resumen puntual, véase Peters, Anne y Pagotto, Isabella, op. cit., pp. 6-9, de donde obtuvimos parte de esta discusión. También sugerimos Dixon, Martín et. al., op. cit., pp. 48-52, quienes recopilan argumentos de diversos autores sobre el tema. Dos artículos más que enriquecen la discusión: Toro Huerta, Mauricio Iván del, op. cit., pp. 518 y ss., y Cárdenas Castañeda, Fabián Augusto, "A Call for Rethinking the Sources of International Law: Soft Law and the Other Side of the Coin", Anuario Mexicano de Derecho Internacional, vol. XIII, 2013, pp. 376 y ss. Finalmente, para una distinción entre las normas y las no normas, lo no legal y lo legal, o lo prelegal y lo legal, véase Weil, Prosper, "Towards Relative Normativity in International Law?, The American fournal of International Law, vol. 77, núm. 3, 1983, pp. 415 y ss.

30 Peters, Anne y Pagotto, Isabella, op. cit., pp. 9-12.

31 La expresión "normas no legales" se ha utilizado en el régimen de control de armamento. Para más detalles, véase Williamson, Richard L. Jr., "Hard Law, Soft Law, and Non-Law in Multilateral Arms Control: Some Compliance Hypotheses", Chicago fournal of International Law, vol. 4, núm. 1, 2003, pp. 62 y 63.

32 Cárdenas Castañeda, Fabián Augusto, op. cit., pp. 382 y ss. 
bajo las reglas de la CV de 1969, sin que se formule una obligación directa: se trata de normas vagas, ambiguas, que no tienen aplicabilidad directa de hacer, dejar hacer o no hacer. Éstas se constituyen en preceptos de aspiración (metas aspiracionales), escritas de manera abstracta en su máxima expresión; a su vez, crean propósitos amplios y generales, no específicos. ${ }^{33}$ Si la norma aparece aislada o desconectada de otras normas del instrumento, no tendrá implicación jurídica y se estará ante el non-legal soft law. Cuando el precepto se localiza en alguna parte específica del tratado para dar sentido a todo o a una parte del cuerpo normativo, y se redacta como paradigma o lineamiento para interpretar otras normas del mismo, podrá ser considerado legal soft law. Los instrumentos no vinculantes, como declaraciones políticas, lineamientos, reportes de organizaciones internacionales u organismos no internacionales, son non-legal soft law. ${ }^{34}$

Se advierte, entonces, que la terminología y tipo de normas que designa la expresión soft law serán tan diversos como sean las visiones o planteamientos de los autores que aborden el tema. Por ello, para los fines de este trabajo podemos elaborar algunas reflexiones según lo examinado en párrafos anteriores, sin pretender hacer una generalización conceptual ni sugerir una tendencia doctrinal.

Primero, los tratados son acuerdos internacionales cuyo contenido normativo es, en principio, de naturaleza jurídico-vinculante o de hard law. Priva la idea de la coacción o exigibilidad en el cumplimiento de las normas, independientemente de que exista o no, o de que esté claramente identificada o no, una sanción, la naturaleza de la obligación misma, o el mecanismo para hacer efectivo el cumplimiento de una norma o una sentencia. Es relevante que los Estados acepten (perciban y sientan) que una norma representa una obligación, y que su cumplimiento es requerido y no meramente opcional. Coincidimos en que algunas normas de hard law pueden ser más o menos rigurosas que otras, pero en definitiva la obligatoriedad las caracteriza: todas producen efectos jurídicos.

Segundo, aceptamos que los tratados pueden contener preceptos de naturaleza distinta a las normas de hard law, y que la mejor forma de des-

33 En sentido estricto, estas normas podrían ser vinculantes por sí mismas, no obstante el hecho de que no tengan implicaciones directas. Para comprender el significado de esto, ibidem, p. 383.

34 Para un significado parecido de estas dos expresiones, acúdase a Chinkin, C. M., op. cit., pp. 851 y ss. 
cribirlos es como normas de derecho suave, blando o flexible, esto es, normas de soft law. Independientemente de que estos preceptos se consideren jurídicos o no (ergo tengan naturaleza vinculante o no desde la visión dicotómica), o que sean graduados o relativos (ergo tengan naturaleza más o menos vinculante desde la visión continua), son normas que carecen de obligatoriedad. Esto no quiere decir que no tengan relevancia jurídica: sí la tienen, aunque en definitiva no son vinculantes. Esta característica es lo que precisamente las diferencia de las normas de hard law, por lo que las normas de soft law no pueden ser menos vinculantes o casi vinculantes. Es verdad que en muchas ocasiones el soft law es el antecedente inmediato del hard law, pero mientras no adquiera tal estatus, simplemente no es hard law. En consecuencia, las normas podrán ser más soft que otras o, incluso, menos soft, pero al fin y al cabo soft law, o sea, no vinculantes. De otro modo, ¿para qué la diferenciación doctrinal entre lo hard y lo soft?

Tercero, y no obstante lo anterior, admitimos, como lo hacen otros doctrinarios, que no es fácil distinguir cuándo una norma en un tratado es de hard law y cuándo de soft law (o cuándo es más rigurosa o cuándo es menos suave). Desde luego, hay que atender al origen y características del precepto mismo (es decir, intencionalidad, negociación, redacción, lenguaje, precisión, generalidad, etcétera), y al hecho de sentirse obligado por una norma, pero no siempre existe la nitidez normativa que se quisiera para lograr tal diferenciación.

Finalmente, y como lo veremos en los próximos apartados, nos sumamos al pronunciamiento doctrinal de que el soft law se ha manifestado de manera clara en el derecho internacional ambiental y, por ende, en el régimen climático. Es común la existencia de normas de soft law en instrumentos internacionales de hard law.

\section{EL SOFT LAW EN EL DERECHO INTERNACIONAL AMBIENTAL}

Cuna principal del soft law es el derecho internacional relativo al régimen de protección del ambiente, es decir, el derecho internacional ambiental. ${ }^{35}$ Es aquí donde el soft law se ha constituido en un "fenómeno dominante", ${ }^{36}$

35 Ejemplos histórico-doctrinales que sustentan esta afirmación son Dupuy, PierreMarie, op. cit., p. 421, y Kiss, Alexandre Ch., Survey of Current Developments in International Environmental Lawe, Morges, IUCN Environmental Policy and Law Paper, 1976, p. 17.

36 Cfr. Juste Ruiz, José, Derecho internacional del medio ambiente, Madrid, McGraw-Hill, 1999, p. 47. 
y donde esa "infinita variedad de formas"37 que lo caracteriza se ha expresado de manera constante y diversa. Las razones de su presencia y desarrollo en la rama jurídica aludida se han abordado de manera profusa en la literatura existente, lo que permite comprender los motivos por los que los Estados lo han adoptado en la práctica para regular diversos temas ambientales, el climático incluido. Por ello, es importante distinguir cuándo se estará frente a una norma soft, y cuáles son las explicaciones más relevantes que han esbozado los especialistas sobre su recurrencia. El enfoque que adoptamos en los párrafos siguientes es, desde luego, en relación con aquellas normas que se insertan en los tratados, lo que corresponde en última instancia a la parte esencial de análisis en este artículo.

Comencemos por señalar que es común que los tratados relativos al derecho internacional ambiental incorporen normas, preceptos, disposiciones o, incluso, textos completos de soft law. ${ }^{38}$ Lo anterior, sin dejar de reconocer, al mismo tiempo, la obvia manifestación de este fenómeno a través de instrumentos que carecen de obligatoriedad o a través de normas que aún no se han consolidado formalmente como tales. ${ }^{39}$

Ahora bien, distinguir cuándo una norma convencional es de naturaleza soft, vis à vis una de naturaleza hard, no es tarea fácil de realizar. ${ }^{40}$

37 La expresión entrecomillada se ha vuelto un tanto famosa a partir del artículo de Baxter, R. R., op. cit.

38 Esto se observa de manera clara, por ejemplo, en Bodansky, Daniel, op. cit., p. 104; Dupuy, Pierre-Marie, op. cit., p. 430; Guruswamy, Lakshman, International Environmental Law, 2a. ed., Thomson-West, 2003, p. 27; Juste Ruiz, José, op. cit., p. 47; Kiss, Alexandre Ch. y Shelton, Dinah, International Environmental Law, 3a. ed., Nueva York, Trasnational Publishers, 2004, p. 73; Maljean-Dubois, Sandrine, "The Making of International Law Challenging Environmental Protection", en Kerbrat, Yann y Maljean-Dubois, Sandrine (eds.), The Transformation of International Environmental Law, Oxford-París, A. Pedone \& Hart, 2011, p. 46; Rey Caro, ErnestoJ., Temas de derecho internacional ambiental, Córdoba (Argentina), Marcos Lerner Editora Córdoba, 1998, p. 18. Cada autor realiza las especificaciones pertinentes sobre lo que para ellos significa el soft law.

39 Para efectos didácticos, el soft law se manifiesta in genere a través de: i) instrumentos que no tienen per se fuerza jurídica vinculante; ii) normas que se establecen en instrumentos hard, y iii) normas en proceso de gestación, es decir, normas que no han entrado en vigor o aún no se cristalizan como tales. Véase Nava Escudero, César, "Guía mínima para la enseñanza del derecho internacional ambiental en México", Boletín Mexicano de Derecho Comparado, nueva serie, año XXXVIII, núm. 113, mayo-agosto de 2005, p. 822.

40 El problema de la distinción entre derecho suave y derecho duro en instrumentos internacionales, de donde se infiere la problemática para las normas en tratados, ocupa un lugar de discusión desde hace algún tiempo en la literatura relativa a lo internacional 
Por ello, se ha sugerido que ante todo se examine para su identificación el contenido mismo del precepto en cuestión, que sea caso por caso, y que se atienda a diversos factores o variables que estén relacionados, por ejemplo, con el origen mismo del texto convencional y con las condiciones formales y políticas de su adopción. ${ }^{41}$ En este sentido, hay que tomar en cuenta para cada norma analizada, en su caso, el tipo de conducta o comportamiento que se espera o se requiere de los Estados parte en un tratado. Esto conducirá, eventualmente, a reconocer una norma de soft law.

¿Cuántos y cuáles podrían ser los criterios para la identificación de la normativa soft? Bueno, como es de suponer, no hay un número exacto ni tampoco una tipología uniforme. Sin embargo, podemos señalar que uno de estos criterios es la dimensión del lenguaje que se emplea en una determinada disposición convencional. En efecto, el lenguaje (o, incluso, la redacción) pueden revelar la existencia de una norma de soft law, como sucede - por ejemplo - con enunciados que son exhortativos o meramente "aspiracionales" ${ }^{42}$ sin ser vinculantes en lo absoluto. ${ }^{43}$ En este caso, se diría en idioma inglés que estamos frente a normas internacionales de comportamiento hortatory o aspirational. ${ }^{44}$

El lenguaje soft puede manifestarse de formas distintas, pero una de las más recurrentes es a través de ciertos verbos ${ }^{45}$ tales como "deberían", "podrán" o "recomendarán", en contraposición a aquéllos utilizados en normas hard, como "deberán" o "requerirán". ${ }^{46}$ Otros verbos de soft law pueden ser "pedir", "solicitar", "invitar", "alentar" o "exhortar", en contraposición con los verbos "ordenar" o "prohibir". ${ }^{47}$ Aunado a lo anterior,

ambiental. Véase, por ejemplo, Cabrera Acevedo, Lucio, El derecho de protección al ambiente, México, UNAM, Instituto de Investigaciones Jurídicas, 1981, pp. 97 y 98.

41 Dupuy, Pierre-Marie, op. cit., pp. 430 y 431.

42 La voz "aspiracional" no está registrada en el Diccionario de la lengua española, pero con ella nos referimos a la declaración o aspiración de alcanzar o lograr algo, es desear o querer conseguir algo.

43 Para una afirmación de este tipo, véase Guruswamy, Lakshman, op. cit., p. 27.

44 Williamson, Richard L. Jr., op. cit., p. 63.

45 Para un ejemplo bastante completo de esto, véase Bodansky, Daniel, op. cit., pp. 103 y 104. En nuestro idioma puede consultarse Juste Ruiz, José, op. cit., p. 55, en la nota de pie de la p. 61.

46 Respectivamente, en inglés son should, may y recommend, y las que se contraponen son shall y require.

47 Respectivamente, en inglés serán request, ask, invite, call, en contraposición de order o forbid. 
también es recurrente que el lenguaje incluya ciertas frases que hacen que la norma sea imprecisa o vaga; es decir, son frases o expresiones casi sacramentales que relativizan el contenido mismo del precepto y otorgan un amplio margen de acción a los Estados. Los ejemplos más comunes son los siguientes: "según corresponda", "cuando proceda", "cuando corresponda", "cuando sea el caso", "según el caso", "según proceda", "en lo posible", "lo antes posible", "en la medida de lo posible", "en la medida de sus posibilidades", "a la luz de sus circunstancias nacionales", "de interés nacional", entre otros. ${ }^{48}$ En ocasiones, los preceptos imprecisos o vagos se utilizan para crear estándares, más que reglas propiamente; mientras aquéllas dejan que el actuar de los Estados sea discrecional o que quede a su arbitrio, éstas establecen qué conductas son permisibles y cuáles no. En principio, los estándares pueden ser más flexibles o laxos y las reglas más rigurosas, pero se advierte que esto no es necesariamente una constante. ${ }^{49}$

Estos verbos y expresiones se localizan comúnmente en el articulado de los tratados internacionales ambientales — aunque también en sus preámbulos-, manifestándose de forma aleatoria. Por ejemplo, en la Convención Relativa a los Humedales de Importancia Internacional Especialmente como Hábitat de Aves Acuáticas (adoptada en Ramsar, Irán, el 2 de febrero de 1971) se distingue un precepto con expresiones de soft law, tal y como lo hemos especificado con antelación. Se establece en el numeral 2 del artículo 4o. lo siguiente:

Cuando una Parte Contratante por motivos urgentes de interés nacional retire de la Lista o reduzca los límites de un humedal incluido en ella, deberá compensar en la medida de lo posible la pérdida de recursos de humedales y en particular crear nuevas reservas naturales para las aves acuáticas y para la protección

48 En inglés, las frases favoritas son as appropriate, as far as possible e, incluso, as soon as posible, según el contexto de la disposición. En este sentido, se ha señalado que tales expresiones tienen el efecto drástico de mitigar, cuando no de mutilar, cualquier posibilidad de medir la obligación respectiva. Al respecto, consúltese Van Lierop, Robert F. y Székely, Alberto, "Compliance with Environmental Treaties: The Empirical Evidence A Commentary on the Softening of International Environmental Law", Proceedings of the Annual Meeting, vol. 91, 1997, p. 238.

49 Detalles en Bodansky, Daniel, op. cit., pp. 104-106. Sobre los estándares, véase Maljean-Dubois, Sandrine, op. cit., p. 47. 
de un porción adecuada de su hábitat original, en la misma región o en otro lugar. ${ }^{50}$

En este mismo sentido también son ejemplo los encabezados de los artículos para dar a los Estados enorme flexibilidad en el cumplimiento de una norma. Un tratado puede, incluso, reiterar este tipo de expresiones a lo largo de su texto, como es el caso del Convenio sobre la Diversidad Biológica (adoptado en Río de Janeiro, Brasil, el 5 de junio de 1992), donde la frase habitual para varios encabezados en sus primeros artículos es la de "...en la medida de lo posible, y según proceda...". ${ }^{51}$ A continuación señalamos algunos ejemplos (las cursivas son nuestras):

Artículo 5. Cada Parte Contratante, en la medida de lo posible y según proceda, cooperará con otras Partes Contratantes, directamente o, cuando proceda, a través de las organizaciones internacionales competentes, en lo que respecta a las zonas no sujetas a jurisdicción nacional, y en otras cuestiones de interés común para la conservación y la utilización sostenible de la diversidad biológica.

Artículo 7. Identificación y seguimiento. Cada Parte Contratante, en la medida de lo posible y según proceda, en especial para los fines de los artículos 8 a 10...

Artículo 8. Conservación in situ. Cada Parte Contratante, en la medida de lo posible y según proceda...

Artículo 9. Conservación ex situ. Cada Parte Contratante, en la medida de lo posible y según proceda, y principalmente a fin de complementar las medidas in situ...

Otro criterio para identificar normas de soft law es el de ubicar la existencia de principios ambientales (es decir, conocidos como principios fundamentales de derecho internacional ambiental), ${ }^{52}$ que se incorporan

50 Las cursivas son nuestras para enfatizar expresiones amplias e imprecisas. Habrá laxitud en las interpretaciones y explicaciones que cada Estado haga sobre lo que signifique "urgente" e "interés nacional".

51 Los encabezados incluyen los temas de cooperación (artículo 5o.); identificación y seguimiento (artículo 7o.); conservación in situ (artículo 8o.); conservación ex situ (artículo 9o.); utilización sostenible de los componentes de la diversidad biológica (artículo 10); incentivos (artículo 11). Lo anterior, sin perjuicio de que en otras partes del texto (en numeral e inciso) aparezca tal frase.

52 Estos principios se distinguen de los principios generales de derecho internacional; véase Sands, Philippe, Principles of International Environmental Law, 2a. ed., Cambridge, Cambridge University Press, 2003, p. 232. Hace tiempo que la doctrina se refiere a ellos como 
en los preámbulos o en el articulado de los tratados. Es común que estos principios, originariamente plasmados en instrumentos de derecho suave, sigan siendo soft al insertarse en un tratado, particularmente cuando el propio principio en texto convencional se liga a verbos o expresiones como los arriba descritos. Esto sucedió en su momento con el principio de precaución en la CMNUCG (adoptada el 9 de mayo de 1992), la cual incluyó en diversas ocasiones el verbo "deberían", y dejó como potestativa la cooperación entre aquellas partes que estén interesadas para hacer frente al cambio climático. Así lo establece el artículo 3.3 de dicho tratado:

Artículo 3. Principios.

$\cdots$

3. Las Partes deberian tomar medidas de precaución para prever, prevenir o reducir al mínimo las causas del cambio climático y mitigar sus efectos adversos. Cuando haya amenaza de daño grave o irreversible, no debería utilizarse la falta de total certidumbre científica como razón para posponer tales medidas, tomando en cuenta que las políticas y medidas para hacer frente al cambio climático deberían ser eficaces en función de los costos a fin de asegurar beneficios mundiales al menor costo posible. A tal fin, esas políticas y medidas deberían tener en cuenta los distintos contextos socioeconómicos, ser integrales, incluir todas las fuentes, sumideros y depósitos pertinentes de gases de efecto invernadero y abarcar todos los sectores económicos. Los esfuerzos para hacer frente al cambio climático pueden llevarse a cabo en cooperación entre las Partes interesadas. ${ }^{53}$

Algo similar podría ocurrir cuando se incorporan principios generales de derecho al tratado. Es el caso del tema de la responsabilidad y reparación de daño, donde ha llegado a utilizarse un lenguaje en "tono esencialmente exhortatorio, cercano a un pronunciamiento de mera lege ferenda". ${ }^{54}$

No obstante lo anterior, dentro del régimen internacional de protección al ambiente también sucede que algunos principios ambientales son dotados de un significado concreto en los tratados, ${ }^{55}$ lo que eventualmente se traduce en un acercamiento a la dimensión hard. Esto explica, en parte,

\footnotetext{
"principios fundamentales de derecho internacional ambiental". Ejemplos son Juste Ruiz, José, op. cit., p. 47, y Kiss, Alexandre Ch., Survey of Current..., cit., p. 29. Nosotros seguimos esto en Nava Escudero, César, "Guía mínima...”, op. cit., pp. 826 y ss.

53 Las cursivas son nuestras para hacer patente el lenguaje soft.

54 Para más sobre esto, véase Juste Ruiz, José, op. cit., p. 75.

55 Kiss, Alexandre Ch. y Shelton, Dinah, International Environmental..., cit., p. 93.
} 
por qué algunos doctrinarios sostienen que un principio de este tipo pueda llegar a ser vinculante por derecho convencional o por derecho consuetudinario. ${ }^{56}$ Por ejemplo, el principio de precaución (uno de los principios fundamentales del derecho internacional ambiental por excelencia) aparenta o quiere adquirir, al menos en el plano estrictamente regional europeo, el estatus de un principio de derecho consuetudinario, ${ }^{57}$ lo que, sin embargo, no está del todo claro todavía.

Dos cuestiones más. Primero, que la frecuente referencia e incorporación de este tipo de principios en tratados deriva, a su vez, en la formulación y desarrollo de la costumbre. Esto parece haber ocurrido con el principio relativo al derecho soberano que tienen los Estados para explotar sus recursos naturales y el deber de no causar daño al ambiente de otros Estados ni de zonas fuera de su jurisdicción nacional. ${ }^{58} \mathrm{El}$ principio es considerado por la doctrina como derecho consuetudinario y como tal confirmado por la propia Corte Internacional de Justicia (en adelante, CIJ) a partir de $1996 .{ }^{59}$ Segundo, que un principio ambiental consagrado

56 En este sentido, véanse Kiss, Alexandre Ch., Survey of Current..., cit., p. 27; Sands, Philippe, op. cit., p. 232, y Székely, Alberto, "El medio ambiente: derecho internacional", Revista de la Facultad de Derecho de México, México, t. XXVI, núms. 103-104, julio-diciembre de 1976, p. 332.

57 Véase lo que al respecto explica De Sadeleer, Nicolas, "The Precautionary Principle in International Law", en Kerbrat, Yann y Maljean-Dubois, Sandrine (eds.), The Transformation of International Environmental Law, Oxford-París, A. Pedone \& Hart, 2011, p. 88.

58 Este principio (que recoge el principio de soberanía de los Estados sobre sus recursos naturales y el principio de prevención del daño ambiental) se consagró en la Declaración de Estocolmo de 1972, que a la letra dice: "Principio 21. De conformidad con la Carta de las Naciones Unidas y con los compromisos del derecho internacional, los Estados tienen el derecho soberano de explotar sus propios recursos en aplicación de su propia política ambiental y la obligación de asegurar que las actividades que se lleven a cabo dentro de su jurisdicción o bajo su control no perjudiquen al medio de otros Estados o de zonas situadas fuera de toda jurisdicción nacional". El texto fue incorporado casi íntegro como principio 2 en la Declaración de Río de 1992. Los antecedentes de dicho principio son la sentencia arbitral en el asunto de la Fundición de Trail de 1941, la sentencia del Tribunal Internacional de Justicia en el asunto del Estrecho de Corfú de 1949, y la sentencia arbitral en el asunto del Lago Lanós de 1957. Para mayor detalle, acúdase a Juste Ruiz, José, op. cit., pp. 72 y ss.; Sands, Philippe, op. cit., pp. 236 y ss., y Székely, Alberto y Ponce-Nava, Diana, "La Declaración de Río y el derecho internacional ambiental", en Glender, Alberto y Lichtinger, Víctor, La diplomacia ambiental. México y la Conferencia de las Naciones Unidas sobre Medio Ambiente y Desarrollo, México, Secretaría de Relaciones Exteriores-Fondo de Cultura Económica, 1994, pp. 310 y 311.

59 Más detalles en Sands, Philippe, op. cit., p. 236. 
en un tratado como norma puede convertirse en un principio general del derecho, como a los que se refiere el artículo 38 del Estatuto de la CIJ, y de aquí seguir la suerte jurídica que tienen estos principios frente y respecto a otras fuentes del derecho internacional. Es el caso del principio relativo a que los Estados tienen la obligación general de proteger y preservar el medio marino, según el artículo 192 de la Convención de las Naciones Unidas sobre el Derecho del Mar (adoptada en Montego Bay, Jamaica, en 1982). ${ }^{60}$

Independientemente de la existencia de otros criterios que pudieran ser útiles en la identificación de normas de soft law en los tratados (como detectar la falta de mecanismos normativos o institucionales para el cumplimiento de un precepto convencional determinado), es relevante referirnos a dos de las razones más importantes por las que los Estados optan por este tipo de normas. En primer lugar, la propia flexibilidad o elasticidad de la norma de soft law permite a los Estados alcanzar cierto consenso respecto a un asunto ambiental, lo cual no se lograría si la naturaleza misma del precepto fuera muy rigurosa. Esto se explica en parte porque los Estados pueden tener intereses políticos y socioeconómicos muy divergentes sobre un tema, que les impidan llegar a un acuerdo en relación con la adopción de una obligación ambiental común. ${ }^{61}$ Entre más vaga, imprecisa, genérica o relativizada se encuentre la norma, el Estado podrá adecuarse con mayor facilidad a ella, sin la presión del cumplimiento forzoso de la misma o de la posibilidad de ser sancionado por incumplimiento.

En segundo lugar, la norma de soft law es una herramienta muy útil que supone (aunque no infaliblemente) un escenario donde todos los Estados y el objetivo del tratado en cuestión puedan beneficiarse (win-win scenario), al menos en términos temporales y con la expectativa de que así será. En efecto, el derecho suave convencional destraba no sólo aspectos de difícil consenso entre las partes, sino también cuestiones relativas a conductas o resultados que supondrían una respuesta rápida o sujeta a plazos muy específicos, pero que no siempre es bienvenida. En ocasiones, los Estados prefieren actuar con cierta cautela, a mediano o largo plazos, esperando la cooperación y el financiamiento de otras naciones u organizaciones inter-

60 Ésta es la opinión de Vallarta Marrón, José Luis, op. cit., p. 61. Entendemos que este precepto, como obligación general, es una obligación de comportamiento más que de resultado, y por lo tanto está ligado a una concepción de soft law, tal y como lo hemos explicado en este trabajo.

61 Guruswamy, Lakshman, op. cit., p. 28. 
nacionales; a veces esta conducta es resultado de la naturaleza del problema ambiental a enfrentar. ${ }^{62}$ A su vez, se abre la posibilidad de consolidar procesos que formen hacia el futuro obligaciones de hard law. Recordemos que ante la negativa de ciertos Estados para adoptar compromisos vinculantes en tal o cual sentido en un tratado, un precepto de soft law podría dar la pauta para que su contenido se incluya posteriormente en otros instrumentos. ${ }^{63}$ Así como el soft law puede crear más soft law, también puede crear hard law. Esto contribuye, como ya lo señalamos, a la formación eventual y hacia el futuro del derecho internacional convencional o consuetudinario.

Lo criticable de estas razones es que no representan en lo absoluto la mejor respuesta que plantea un problema ambiental de características globales para un accionar concreto, rápido y específico de los Estados. Si bien las normas de soft law en los tratados sirven para consolidar principios básicos ambientales, guiar o dirigir el comportamiento y conducta de las partes contratantes, y constituirse como un antecedente del derecho duro, también son utilizadas para enmascarar desacuerdos interestatales y la falta de compromisos verdaderos de algunos Estados para enfrentar conjuntamente dilemas de la mayor trascendencia. Conducirse de manera laxa, posponer decisiones, o comportarse de tal o cual manera en la medida en la que otros se comporten sin sentirse obligados a ello, es característico de la norma convencional en el derecho internacional ambiental.

No debe sorprender que en cualquiera de los regímenes internacionales de protección al ambiente los tratados desarrollen tales contenidos. El derecho suave es simple y sencillamente un proceso de creación de normas bastante común dentro de lo jurídico internacional ambiental. Los Estados acuden reiteradamente a él al momento de negociar y redactar los textos respectivos. En consecuencia, y aún sin considerarlo trivial o superfluo, este proceso no siempre ofrece el mejor de los escenarios para responder a los grandes retos ambientales.

62 En efecto, hay ocasiones que la naturaleza misma del problema ambiental hace casi imposible definir el contenido exacto de una obligación, como cuando ocurre con asuntos relacionados con la contaminación de largo alcance o a largo plazo. Para esto, véase Kiss, Alexandre Ch. y Shelton, Dinah, International Environmental..., cit., p. 95.

63 Véase lo señalado en Shelton, Dinah, op. cit., pp. 63 y 64. 


\section{EL AGUERDO DE PARÍS: REINGIDENGIA DEL SOFT LAW EN EL RÉGIMEN CLIMÁTICO}

Empecemos por aclarar que el régimen climático pertenece a foros propiamente ambientales, es decir, es uno de los diversos regímenes internacionales de protección al ambiente. ${ }^{64}$ En efecto, ya desde hace algunos años la doctrina lo ha incluido como un sector o capítulo dentro del derecho internacional ambiental. ${ }^{65} \mathrm{Y}$ desde el punto de vista normativo, este régimen se ha caracterizado por tener un desarrollo normativo ambiental altamente orientado al soft law. Esto se refleja en mayor o menor medida en los tres tratados más importantes en la materia: la CMNUCC de 1992, el PK de 1997 y el AP de 2015.

\section{La Convención Marco de las Naciones Unidas sobre el Cambio Climático}

Como ha sucedido con otros regímenes internacionales ambientales, el del cambio climático tuvo su inicio y preliminar desarrollo con reuniones y conferencias, ${ }^{66}$ que derivaron en una serie de declaraciones y otros instrumentos de naturaleza soft law. Al adquirir decidida relevancia internacional a finales de la década de los ochenta del siglo pasado, el fenómeno del cambio climático fue objeto de una serie de evaluaciones científicas y técnicas del órgano intergubernamental más importante creado ex profeso para este tema: el Grupo Intergubernamental de Expertos sobre el Cambio Climático, también conocido como Panel Intergubernamental de Cambio Climático (en adelante, PICG). Establecido en 1988 por la Organización Meteorológica Mundial y el Programa de las Naciones Unidas para el Medio Ambiente, el PICG publicó en 1990 un primer informe de evaluación que fue, entre otros, fundamental para el inicio de las negociaciones en la elaboración de un tratado bajo el auspicio de las Naciones

64 Por ejemplo, Borrás Pentinat, Susana, Los regímenes internacionales de protección del medio ambiente, Valencia, Tirant lo Blanch, 2011.

65 Prácticamente, todos los libros sobre derecho internacional ambiental consultados dedican un espacio considerable al tema. También es común verlo como capítulo o apartado en los libros de derecho ambiental.

66 Destacan durante este periodo la I Conferencia Mundial sobre el Clima en Ginebra, Suiza, 1979; las reuniones en Villach, Austria, 1985; Bruselas, Bélgica, 1986; Toronto, Canadá, 1988; Ottawa, Canadá, 1989; La Haya, Países Bajos, 1989, y la II Conferencia Mundial sobre el Clima otra vez en Ginebra, Suiza, 1990. 
Unidas, lo que ocurriría en 1990 con base en la Resolución 45/212 de la Asamblea General. ${ }^{67} \mathrm{El}$ resultado de los trabajos fue la creación de la CMNUCG, en vigor desde el 21 de marzo de 1994.

Aunque la CMNUCC representa el primer acuerdo internacional ambiental vinculante sobre el clima, su contenido normativo es predominantemente de derecho suave. ${ }^{68} \mathrm{Si}$ bien se establecen algunas obligaciones concretas para las partes contratantes, el texto es un ir y venir de disposiciones con lenguaje genérico, impreciso, vago o relativizado. Un ejemplo de esto es el que se refiere a las concentraciones de gases de efecto invernadero (en adelante, GEI) en la atmósfera, que es lo que está produciendo el cambio climático. El objetivo último de la CMNUCG es lograr la estabilización de tales concentraciones "a un nivel que impida interferencias antropógenas peligrosas", pero no se precisa cuál debe ser tal nivel ni en qué tiempo hacerlo, ${ }^{69}$ amén de utilizar el vocablo "debería" en el texto de referencia, lo que es característico en un enunciado de soft law. ${ }^{70}$

Otro ejemplo son los enunciados relativos a la incorporación de diversos principios fundamentales de derecho internacional ambiental, como los de equidad intergeneracional, responsabilidades comunes pero diferenciadas, o de precaución. Independientemente de que en todos ellos se hace un uso extenso de la palabra "deberían", el encabezado del precepto donde están incluidos (artículo 3o.) se refiere a ellos como meras guías y no como reglas vinculantes. ${ }^{71}$

Aunado a lo anterior, el importante artículo relativo a los compromisos para todas las partes contratantes (artículo 4o.) tiene un encabezado que sujeta tales compromisos a las prioridades nacionales y regionales de desa-

67 Más detalles sobre esto en Birnie, Patricia et al., International Law and the Environment, 3a. ed., Oxford, Oxford University Press, 2009, p. 356, y Sands, Philippe, op. cit., pp. 358 y 359 .

68 Esto puede constatarse, por ejemplo, en Juste Ruiz, José, op. cit., p. 290.

69 Véase lo que también se señala en Birnie, Patricia et al., op. cit., p. 358.

70 El artículo 2o. respectivo señala: "El objetivo último de la presente Convención... es lograr... la estabilización de las concentraciones de gases de efecto invernadero en la atmósfera a un nivel que impida interferencias antropógenas peligrosas en el sistema climático. Ese nivel debería lograrse en un plazo suficiente para permitir que los ecosistemas se adapten naturalmente al cambio climático" (las cursivas son nuestras).

71 De esta opinión es Birnie, Patricia et al., op. cit., p. 359. El encabezado en cuestión señala: "Las Partes, en las medidas que adopten para lograr el objetivo de la Convención y aplicar sus disposiciones, se guiarán, entre otras cosas, por lo siguiente...", y luego enumera los principios. 
rrollo, y a los objetivos y circunstancias de cada una de ellas. El contenido mismo de cada compromiso está redactado, igualmente, de manera que difícilmente pudiera pensarse en disposiciones rigurosas, con todo y que en el propio artículo 4.2, incisos $a$ y $b$, se establezca que las partes que son países desarrollados y las incluidas en el Anexo I se comprometen específicamente a regresar sus emisiones a los niveles de 1990 antes del año $2000 .{ }^{72}$ De aquí que a estos preceptos se les haya correctamente caracterizado de obligaciones mínimas, lo que ha conducido a decir que este tratado "establece un régimen particularmente confuso de compromisos a cargo de las Partes, en el que la ambigüedad es la característica dominante...". ${ }^{73}$

Advertimos que el contenido de soft law en la CMNUCG se explica en gran medida porque fue un instrumento adoptado como convención marco. Este tipo de tratados, propios para la regulación de temas ambientales regionales o globales, establecen obligaciones generales, crean arreglos institucionales básicos y determinan procedimientos para la adopción futura de obligaciones más precisas en protocolos subsecuentes. ${ }^{74}$ Por tanto, no sorprende que este tratado tenga un alto contenido de normativa suave. Más allá de que esto mismo haya servido para cuestionar moralmente si tal formato jurídico fue el adecuado para enfrentar el cambio climático, ${ }^{75}$ lo cierto es que los Estados así lo decidieron.

\section{El Protocolo de Kyoto a la CMNUCC}

Dentro de los arreglos institucionales de la CMNUGC se creó, entre otros, la Conferencia de las Partes (en adelante, COP) en calidad de órga-

72 Más detalles en Birnie, Patricia et al., op. cit., pp. 359 y 360. Las partes incluidas en el Anexo I (es decir, países miembros de la OCDE, la Comunidad Europea y países en proceso de transición a una economía de mercado) tienen ciertos compromisos específicos, pero aún así son imprecisos, como el que no exista cuantificación sobre la limitación de las emisiones de GEI.

73 Y es que en el artículo se establecen distinciones, categorías entre las partes, y se desarrolla un régimen asimétrico de obligaciones; en suma, diversifica, condiciona y matiza los compromisos establecidos. Véase lo señalado por Juste Ruiz, José, op. cit., pp. 291 y 292, y para la cita textual, p. 296.

74 Kiss, Alexandre Ch. y Shelton, Dinah, International Environmental..., cit., p. 78; Sands, Philippe, op. cit., p. 128.

75 Para una excelente discusión sobre la idoneidad moral de la GMNUCG, acúdase a Garvey, James, The Ethics of Climate Change. Right and Wrong in a Warming World, Londres, Continuum, 2008, pp. 114-120. 
no supremo de la propia Convención. A ésta corresponde, según el artículo 7o., examinar la aplicación del tratado y de todo instrumento jurídico conexo que adopte, así como tomar - conforme a su mandato- las decisiones necesarias para promover la aplicación eficaz del mismo. Entre sus diversas competencias se determinó que la COP podía aprobar protocolos (según el artículo 17), lo que habría de suceder durante su tercer periodo de sesiones, en el que se adoptó en Kyoto, Japón, el PK. ${ }^{76}$

En el nuevo instrumento se incluyeron, ahora sí de manera más palpable, normas de hard law, lo que no significó que se dejaran de insertar enunciados soft law. Por un lado, el derecho duro se manifestó, entre otros, a través de obligaciones vinculantes para los países Anexo I de la CMNUCG relativas a compromisos cuantificados de limitación y reducción de emisiones para un primer periodo específico: del 2008 al 2012. Las partes contratantes obligadas - según el artículo 3.1 - habrían de reducir, ya sea de manera individual o conjuntamente, el total de sus emisiones de un número determinado de gases (enumerados en el Anexo "A" del PK) a un nivel inferior en no menos de 5\%, tomando como referencia los niveles de emisión de 1990.

Por el otro, el derecho suave se plasmó al establecer, una vez más, obligaciones generales o flexibles para las partes contratantes. Esto se observa con claridad en relación con las políticas y medidas que habrían de aplicar o seguir elaborando las partes Anexo I, según el artículo 2.1, el cual señala que con el fin de promover el desarrollo sustentable, cada una de ellas al cumplir con sus compromisos cuantificados contraídos "...aplicará y/o seguirá elaborando políticas y medidas de conformidad con sus circunstancias nacionales...". ${ }^{77}$

76 Las negociaciones del PK tuvieron como base el Mandato de Berlín de 1995, adoptado en la primera COP de ese año, y se estableció que los compromisos del artículo 4.2, incisos $a$ y $b$, no eran adecuados, y por tanto era necesario fortalecerlos, entre otras, a través de una limitación cuantificada de sus emisiones en períodos específicamente establecidos. En la siguiente conferencia, en la COP-2, celebrada en Ginebra, Suiza, en 1996, se adoptó una Declaración ministerial, que también sirvió de base al PK, puesto que en ella se instó a los participantes respectivos a acelerar las negociaciones para la adopción de un instrumento que incorporara los preceptos antes mencionados. Los detalles en Sands, Philippe, op. cit., pp. 369 y 370 .

77 La redacción de tal enunciado se debió a la resistencia de Estados Unidos, Canadá, Australia y otras partes Anexo I, que prefirieron un enfoque flexible en esta cuestión en particular, tal y como nos lo recuerda Sands, Philippe, op. cit., p. 372. Las cursivas en la cita textual del artículo son nuestras. 
Otro ejemplo lo constituye el hecho de que no se hubieran establecido compromisos cuantificados de reducción para los países no desarrollados: ninguno de ellos se incluyó en el Anexo "B" del Protocolo. ${ }^{78}$ Esto significa, en realidad, que a estos países no se les requiere de otra cosa que no sea la de cumplir con sus obligaciones ya existentes bajo el artículo 4.1 de la CMNUCG. ${ }^{79}$

Aunado a lo anterior, se incluyeron enunciados que dejan para futuras negociaciones ciertas reglas específicas, guías y metodologías necesarias para la implementación del Protocolo, lo que habría de cristalizarse años más tarde en instrumentos como los Acuerdos de Bonn y los Acuerdos de Marrakech, ambos de 2001 para tales efectos. ${ }^{80}$ Esto sucedió en lo particular con los procedimientos en caso de incumplimiento, según lo establecido en el artículo 18 del PK:

En su primer período de sesiones, la Conferencia de las Partes en calidad de reunión de las Partes en el presente Protocolo aprobará unos procedimientos y mecanismos apropiados y eficaces para determinar y abordar los casos de incumplimiento de las disposiciones del presente Protocolo, incluso mediante la preparación de una lista indicativa de consecuencias, teniendo en cuenta la causa, el tipo, el grado y la frecuencia del incumplimiento. Todo procedimiento o mecanismo que se cree en virtud del presente artículo y prevea consecuencias de carácter vinculante será aprobado por medio de una enmienda al presente Protocolo.

Fue la COP la que adoptó posteriormente un texto para definir los procedimientos y mecanismos en cuestión, y propuso que esto mismo se hiciera en la primera Reunión de las Partes COP11/CMP1 (celebrada en 2005 en Montreal, Canadá), cuestión que derivó, sin embargo, en la adopción mutatis mutandis del texto antes presentado. Sin revisión del protocolo, los preceptos relacionados con el procedimiento descansaron, entonces, en una mera decisión de la reunión de las partes, por lo que no habría tenido, en principio, consecuencias vinculantes. ${ }^{81}$

78 En este Anexo figuran las partes con compromisos cuantificados de limitación o reducción de emisiones.

79 Birnie, Patricia et al., op. cit., p. 361.

80 Véase lo señalado sobre esta cuestión en Sands, Philippe, op. cit., p. 370.

81 Para esta explicación y sobre los alcances vinculantes o no de este texto por las "sanciones" impuestas a Grecia ante los problemas metodológicos en su inventario de GEI, véase Maljean-Dubois, Sandrine, op. cit., p. 49. 
Todo el aparato de hard law/soft law logrado en la GMNUCG, en el PK, así como en sus reuniones y conferencias posteriores, no fueron suficientes para evitar la crítica sobre sus alcances reales en el combate al cambio climático. Si bien algunos sectores importantes de la comunidad internacional y no pocos científicos aplaudieron los esfuerzos logrados, otros más consideraron al marco jurídico climático como "un avance simplemente simbólico", ${ }^{82}$ y en particular al PK como un instrumento más dañino que benéfico. ${ }^{83} \mathrm{Y}$ es que el objetivo de reducción de emisiones resultó ser muy bajo para lo que se esperaba en ciertos escenarios del calentamiento global ${ }^{84}$ lo que aunado a la falta de reducciones cuantificadas vinculantes de países no desarrollados - es decir, discriminación positiva o benigna en su favor-, ${ }^{85} \mathrm{y}$ a la información científica publicada posteriormente a la adopción el PK, se concluyó que el régimen acordado era francamente inadecuado. ${ }^{86}$

En años recientes, la crítica se ha centrado en que los supuestos "estancamientos" o "reducciones" de emisiones de países desarrollados se deben al comercio internacional de traslado de producción sucia a países no desarrollados, ${ }^{87}$ y al proceso de compensación que aquéllos tienen para alcanzar la reducción requerida a través de mecanismos flexibles en el PK (es decir, aplicación conjunta, desarrollo limpio y comercio de derechos de emisión), ${ }^{88}$ mismos que por su naturaleza se asemejan más a enunciados de soft law que de hard law.

$82 \mathrm{Al}$ respecto, véase Antal, Edit, Cambio climático: desacuerdo entre Estados Unidos y Europa, México, Plaza y Valdés-UNAM, Centro de Investigaciones sobre América del Norte (CISAN), 2004, pp. 78 y ss.

83 Véase lo señalado en Beyerlin, Ulrich y Marauhn, Thilo, International Environmental Law, Oxford, Hart-CH Beck-Nomos, 2011, p. 163.

84 Por ejemplo, la reducción para el periodo 2008-2012 de un promedio de 5.2\% entre los países desarrollados contrasta con la necesidad de reducción de entre un $50 \%$ y $80 \%$ para enfrentar los efectos más catastróficos identificados. Borrás Pentinat, Susana, op. cit., pp. 136 y 137. En otro ejemplo, los resultados de la implementación absoluta del PK habrán sido marginales, con una reducción de la temperatura en $0.15 \%$, al compararse con un escenario donde no existiera reducción de bióxido de carbono (business as usual). Guruswamy, Lakshman, op. cit., pp. 218 y ss.

85 Beyerlin, Ulrich y Marauhn, Thilo, op. cit., p. 161.

86 Birnie, Patricia et al., op. cit., p. 371.

87 Esta crítica la tenemos en Klein, Naomi, Esto lo cambio todo. El capitalismo contra el clima, trad. de Albino Santos Mosquera, Barcelona, Paidós, 2015, p. 108.

88 Beyerlin, Ulrich y Marauhn, Thilo, op. cit., pp. 162 y ss. En lo particular, se ha cuestionado la efectividad de los mecanismos de desarrollo limpio por lo que toca a los primeros 
Después de todo, en el último informe del PICG - el Quinto Informe de Evaluación - se mencionó que según los inventarios nacionales de GEI de 2012 presentados a la CMNUCG en octubre de 2013, "las Partes incluidas en el anexo B que habían adoptado compromisos de limitación (y reducción de las emisiones) cuantificadas podían haber mejorado su meta conjunta de reducción de las emisiones durante el primer período de compromiso...". ${ }^{89}$ En este mismo informe se dio a conocer que la conclusión final sobre el cumplimiento de las partes Anexo "B" estaba, en octubre de 2014, todavía en proceso de examen en el marco del PK.

\section{El Acuerdo de París}

Con elogios y críticas, lo plasmado en el PK y lo establecido en las decisiones tomadas por subsecuentes conferencias y reuniones de las partes fueron resultado de arduas negociaciones y de consensos y disensos entre los Estados. Conscientes de que el primer periodo de compromisos expiraría en 2012, la comunidad internacional comenzó, poco después de que el PK entrara en vigor (febrero de 2005), las discusiones para llegar a un acuerdo que modificara o, en su caso, sustituyera al PK para fortalecer el régimen climático. ${ }^{90}$ Las primeras expectativas de que esto ocurriera se centraron tanto en la COP-15 en Copenhague, en 2009, como en la COP16 en Cancún, en 2010, ${ }^{91}$ pero en ninguna de ellas se alcanzó un instrumento vinculante para un periodo posterior a $2012 .{ }^{92}$

$\mathrm{Al}$ poco tiempo, y ante la falta de consenso para lograr un acuerdo vinculante, se adoptó en Doha, Qatar, en diciembre de 2012, en la COP-

años de aplicación. Para más detalles, véase IPCG, Cambio climático 2014: Informe de sintesis. Contribución de los Grupos de trabajo I, II y III al Quinto Informe de Evaluación del Grupo Intergubernamental de Expertos sobre el Cambio Climático, Ginebra, IPCG, 2015, p. 115.

89 IPCG, op. cit., pp. 114 y 115.

90 Beyerlin, Ulrich y Marauhn, Thilo, op. cit., p. 148. Detalles sobre lo que habría de ocurrir de 2007 en la COP-13/CMP-3 en Bali, Indonesia, hasta 2014 en la COP-20/ CMP-10 en Lima, Perú, se encuentran en Flores Liera, Socorro, "El cambio climático, ¿un reto que rebasa a la comunidad internacional?, en González G., Guadalupe et. al. (eds.), México y el multilateralismo del siglo XXI. Reflexiones a los 70 años de la ONU, México, Siglo Veintiuno Editores-ITAM-Senado de la República, 2015, pp. 372 y ss.

91 En este sentido, véase lo señalado en Borrás Pentinat, Susana, op. cit., p. 144.

92 Nava Escudero, César, "México ante la COP-16. Entre la simulación climática y la incertidumbre jurídica", Revista del Posgrado en Derecho de la UNAM, núm. 13, julio-diciembre de 2011 , pp. 212 y ss. 
18/CMP8, la Enmienda de Doha al PK, que - entre otras cosas - estableció: un segundo periodo de compromisos por ocho años (2013-2020); la continuidad de los mecanismos flexibles del PK; nuevos compromisos cuantificados de limitación o reducción de emisiones para el segundo periodo (en Anexo "B"); el compromiso de las partes Anexo I para reducir el total de sus emisiones "a un nivel inferior en no menos del $18 \%$ al de 1990 en el período de compromiso comprendido entre los años 2013 y 2020" (artículo 3.1 bis), etcétera. Algo muy importante de dicha conferencia/reunión fue que se fijó un calendario internacional para alcanzar un acuerdo mundial en 2015, lo que sucedió hacia finales de ese año en París, Francia.

En cierta medida, y con algunas diferencias, el AP confirma y repite lo que ha ocurrido en el régimen climático cuando se hizo la adopción de la CMNUGG y el PK: se trata de un instrumento de hard law con un alto contenido de normas de soft law. Esta reincidencia jurídico-ambiental-climática se ha expresado de maneras diversas en el texto aprobado: algunas de obvia inserción y otras como resultado de una negociación, para que los Estados participantes lograran un acuerdo vinculante y pudieran responder así a las demandas formuladas por diversos actores de la propia comunidad internacional y de la sociedad en general. Muchas de las críticas han estado dirigidas, precisamente, a la parte del soft law de este tratado.

Comencemos, como es lógico, por el preámbulo, el cual contiene un caudal de declaraciones políticas y de buena voluntad, que en el marco de la respuesta al cambio climático, se encuentran asociadas a la erradicación de la pobreza, la seguridad alimentaria, los empleos dignos y los derechos humanos, entre otros. En este sentido, se incluye la importancia de conceptos como los de "la Madre Tierra", la justicia climática, o los estilos de vida y pautas de consumo y producción sustentables. Vinculadas a ellas se da cuenta de que el cambio climático "es un problema de toda la humanidad". Stricto sensu, sin embargo, las críticas al texto del AP no están enfocadas a su preámbulo, sino a su articulado.

Una de las críticas que más ha llamado la atención es aquella que señala que en lugar de que se establecieran contribuciones obligatorias de reducción de emisiones (es decir, metas cuantificadas), se debían plasmar enunciados basados en promesas voluntarias a través de las llamadas "contribuciones determinadas a nivel nacional" (antes en inglés INDC, y ahora 
NDG) ${ }^{93}$ Independientemente de lo poco idóneo de éstas para mantener el aumento de la temperatura mundial muy por debajo de $\operatorname{los} 2^{\circ} \mathrm{C}$ respecto a los niveles preindustriales, según lo establece el artículo 2.1, inciso $a$, del AP, el lenguaje utilizado y la esencia de tales contribuciones es flexible e impreciso, lo que permite suponer que se trata de enunciados de derecho suave. De hecho, todo el sistema en torno a ellas descansa en lo que se ha dado por llamar una "estructura soft". ${ }^{94}$ Si bien las partes deberán de entregar sus contribuciones cada cinco años (lo que aparenta ser de hard law), el texto del tratado las sujeta a una vaga disposición de "progresión a lo largo del tiempo" (artículo 3o. del AP). ${ }^{95}$ La idea de cumplir con el objetivo del "largo plazo" al que se refiere el artículo antes citado se encuentra vinculado al ambiguo, impreciso e indeterminable precepto de que las partes se proponen lograr que las emisiones mundiales de GEI "alcancen su punto máximo lo antes posible” (artículo 4.1). Así, la entrega es de hard law (aunque no es muy rigurosa), y lo que se entrega en contenido (cada país fija sus contribuciones, su nivel de ambición) es de soft law.

93 Los países presentarán tales contribuciones en el marco del AP. Antes de la COP21, el 30 de octubre de 2015 la Secretaría de la CMNUCG publicó un Informe de síntesis sobre el efecto agregado de 119 contribuciones entregadas de 147 Partes (hasta el 1o. de octubre de 2015) como respuesta a la invitación formulada por la COP para tal efecto. Este Informe puede consultarse en United Nations Framework Convention on Climate Change, Synthesis Report on the Aggregate Effect of INDCs, UNFCG, 2015, disponible en: unfccc. int/focus/indc_portal/items/9240.php. Una crítica puntual de antes de la COP-21 en Solón, Pablo, "COP21: A New Disguise for an Old Agreement", noviembre de 2015, disponible en: https://pablosolon.wordpress.com.

94 Así lo describe (soft structure) el profesor Jorge E. Viñuales, director del Cambridge Centre for Environment, Energy and Natural Resource Governance de la Universidad de Cambridge, Inglaterra. Para más detalles sobre las NDG desde su perspectiva, véase Viñuales, Jorge, "The Paris Climate Agreement: An Initial Examination (Part II of III)", EfIL: Talk! Blog of the European Fournal of International Law, 8 de febrero de 2016, disponible en: wrwe.ejiltalk,org/the-pari-climate-agreement-an-initial-examination-part-ii-of-iii/.

95 Las NDC “[son] promesas (pledges) que cada Estado deberá entregar formalmente. El objetivo es que acumuladamente, las medidas nacionales contribuyan a lograr las metas comunes del Acuerdo de París a largo plazo y de manera voluntaria... Es cierto que las contribuciones nacionales deben actualizarse cada cinco años y están sujetas al principio de progresión; es decir, cada promesa renovada será más ambiciosa a lo prometido previamente. Sin embargo, mientras la entrega, comunicación y actualización de las contribuciones nacionales están claramente plasmadas en lenguaje directivo («deberá»), el principio de progresión no lo está (la contribución sucesiva «representará una progresión»)". Rodiles, Alejandro, "El Acuerdo de París: un empujoncito hacia la justicia climática", Nexos, febrero de 2016. 
Alrededor de las NDG se encuentran, además, disposiciones relativizadas. Esto sucede, por ejemplo, con el artículo 6.1 para lograr una mayor ambición en las medidas de mitigación y adaptación. El texto contiene expresiones y palabras soft law que son potestativas y genéricas: "La Partes reconocen que algunas Partes podrán optar por cooperar voluntariamente en la aplicación de sus contribuciones determinadas a nivel nacional para lograr una mayor ambición en sus medidas de mitigación y adaptación y promover el desarrollo sostenible y la integridad ambiental" (las cursivas son nuestras).

Otro aspecto es el relativo a los preceptos sobre la deforestación (y por extensión a los bosques en su función de sumideros) como un asunto primordial para combatir el cambio climático. ${ }^{96} \mathrm{~A}$ lo largo de la COP-21 se alertó sobre la no inclusión o, en su caso, minimización del tema, particularmente por lo que respecta a no insertar en el AP medidas rigurosas para evitar la deforestación. ${ }^{97}$ Lo que quedó plasmado en el artículo 5o., puntos 1 y 2, del AP es un claro ejemplo del derecho suave convencional, donde los enunciados son exhortativos, vagos y con tintes propios de normas no coercibles que reflejan meras expectativas de comportamiento internacional. ${ }^{98}$ Aquí los dos párrafos (las cursivas son nuestras):

\section{Artículo 5}

1. Las Partes deberian adoptar medidas para conservar y aumentar, según corresponda, los sumideros y reservorios de gases de efecto invernadero a que se hace referencia en el artículo 4, párrafo 1 d) de la Convención, incluidos los bosques.

2. Se alienta a las Partes a que adopten medidas para aplicar y apoyar, también mediante los pagos basados en los resultados, el marco establecido en las orientaciones y decisiones pertinentes ya adoptadas en el ámbito de la Convención respecto de los enfoques de política y los incentivos positivos para reducir las emisiones debidas a la deforestación y la degradación de los bosques, y de la función de la conservación, la gestión sostenible de los bosques, y el aumento

96 Sobre este punto en particular, acúdase a Sarasíbar Iriarte, Miren, El derecho forestal ante el cambio climático: las funciones ambientales de los bosques, Navarra, Thomson-Aranzadi, 2007, pp. 169 y ss.

97 Pablo Solón (activista) propuso "un alto en seco a la deforestación" para reducir las emisiones. Más de esto en una nota de Acedo, Alfredo, "Con el Acuerdo de París arderá el planeta", Agencia Latinoamericana de Información (ALAI), 10 de diciembre de 2015, disponible en: wrwe.alainet.org/es/articulo/174215.

98 Esto último constituye una de las formas clásicas de definir el soft law. Véase la clasificación propuesta por Cárdenas Castañeda, Fabián Augusto, op. cit., pp. 376 y ss. 
de las reservas forestales de carbono en los países en desarrollo, así como de los enfoques de política alternativos, como los que combinan la mitigación y la adaptación para la gestión integral y sostenible de los bosques, reafirmando al mismo tiempo la importancia de incentivar, cuando proceda, los beneficios no relacionados con el carbono que se derivan esos enfoques.

Dos cuestiones más de derecho suave apuntan a la normatividad de no cumplimiento y al arreglo de controversias. Como es común que suceda en el derecho internacional ambiental, y por ende en el régimen climático, el AP establece un mecanismo no contencioso para los casos de incumplimiento a través de un comité compuesto por expertos y de carácter facilitador para la aplicación y promoción en el acatamiento de las disposiciones del tratado (artículo 15, puntos 1, 2 y 3). Sobre la cuestión del arreglo de controversias, se aplica mutatis mutandis lo establecido en la CMNUCC, que en su artículo 14 abre la posibilidad para que los Estados acepten, en caso de controversia, la jurisdicción de la CIJ o el arbitraje, pero esto no se ha utilizado hasta el momento. ${ }^{99}$

Finalmente, la preocupación del aumento de la temperatura y su consecuente limitación en menos de $2^{\circ} \mathrm{C}$ se plasmó en una norma meramente aspiracional, y por tanto de soft law. El artículo 2.1, inciso a, del AP señala:

\section{Artículo 2}

1. El presente Acuerdo, al mejorar la aplicación de la Convención [se refiere a la CMNUCG], incluido el logro de su objetivo, tiene por objeto reforzar la respuesta mundial a la amenaza del cambio climático, en el contexto del desarrollo sostenible y de los esfuerzos por erradicar la pobreza, y para ello:

a) Mantener el aumento de la temperatura media mundial muy por debajo de $2^{\circ} \mathrm{C}$ con respecto a los niveles preindustriales, y proseguir los esfuerzos para limitar ese aumento de la temperatura a $1,5^{\circ} \mathrm{C}$ con respecto a los niveles preindustriales, reconociendo que ello reduciría considerablemente los riesgos y los efectos del cambio climático.

Poco abona a estos preceptos de soft law el hecho de que en el Informe de síntesis de la Secretaría de la CMNUCC, relativo al efecto agregado de

99 Para mayor información, véase Viñuales, Jorge, "The Paris Climate Agreement: An Initial Examination (Part III of III)", EfIL: Talk! Blog of the European Fournal of International Law, 8 de febrero de 2016, disponible en: wwre.ejiltalk,org/the-pari-climate-agreement-an-initialexamination-part-iii-of-iii/. 
las contribuciones determinadas a nivel nacional entregadas hasta el 1o. de octubre de 2015, se haya señalado que tales contribuciones sobrepasan el límite de los $2^{\circ} \mathrm{G}$ de temperatura media mundial, dato un tanto alejado del objetivo originario. De hecho, en el documento de decisión de la COP-21 (a la cual se anexa el AP) se reconoce esto en los términos siguientes: "17. Observa con preocupación que los niveles estimados de las emisiones agregadas de gases de efecto invernadero en 2025 y 2030 resultantes de las contribuciones previstas determinadas a nivel nacional no son compatibles con los escenarios de $2^{\circ} \mathrm{C}$ de menor costo...". Además, en dicho documento se señala que para mantener el aumento de la temperatura media mundial por debajo de $2^{\circ} \mathrm{C}$, o incluso por debajo de $1,5^{\circ} \mathrm{C}$, con respecto a niveles preindustriales "se requerirá un esfuerzo de reducción de las emisiones mucho mayor que el que suponen las contribuciones previstas determinadas a nivel nacional". ${ }^{100}$

\section{REFLEXIÓN FINAL}

En el recorrido de análisis sobre la existencia de normas de soft law en los tratados se ha corroborado la tendencia por parte de los Estados, de adoptar dentro del derecho internacional ambiental varios instrumentos de hard law con alto contenido de preceptos no vinculantes. Al pertenecer al régimen internacional de protección al ambiente, el régimen jurídico sobre el clima es una muestra del uso de normas convencionales de derecho suave en instrumentos de derecho duro. El recientemente adoptado AP (diciembre de 2015) permite identificar el predominio de normatividad soft en este

100 Algunas voces críticas sobre tales metas aspiracionales y el contenido del AP señalan, entre otras cosas, lo siguiente: i) “...el acuerdo es una excusa que tienen los políticos para poder decir: tenemos una meta de dos grados e intentaremos hacerlo mejor cada cinco años..." (James Hansen, profesor en la Universidad de Columbia); ii) “.... la meta ambiciosa de la temperatura le faltan los medios ambiciosos para la mitigación... Para llegar al objetivo de $1,5^{\circ} \mathrm{C}$ hay que rebajar las emisiones del $70 \%$ al $95 \%$ a mediados de siglo..." (Steffen Kallbekken, director del Centre for International Climate and Energy Policy del Research Council of Norway), y iii) "el calentamiento causado por las actividades humanas se está acercando ya a un grado y es muy posible que llegue a $1,2^{\circ} \mathrm{C}$ en $2030 \mathrm{con}$ la tendencia actual... Quedarnos en 1, $5^{\circ} \mathrm{C}$ en 2050 va a ser realmente un reto" (Myles Allen, profesor en la Universidad de Oxford). Más sobre esto en un reportaje de Fresneda, Carlos, "El Acuerdo de París: ¿éxito o fraude?", El Mundo, 13 de diciembre de 2015, disponible en: wrore.elmundo.es/ciencia/2015/12/13/566dc1dfee2704ece3d8b4614.html. 
tipo de tratados, tal y como sucedió, si bien con rasgos y matices diferentes, con sus antecesores: la CMNUCG y el PK.

Desde el punto de vista jurídico, no debe adjetivarse como bueno o malo ni como correcto o incorrecto el uso del derecho suave en el formato convencional del régimen climático. Simplemente ha sido una herramienta a la cual han acudido reiteradamente los Estados desde hace tiempo para enfrentar uno de los fenómenos más graves que afecta a la humanidad, y si se permite la expresión, al planeta entero.

Si bien los textos jurídicos climáticos existentes, y en lo particular el AP, no han sido ni serán suficientes para alcanzar los objetivos trazados (mantener muy por debajo de los $2^{\circ} \mathrm{C}$ la temperatura media global y limitar ese aumento a $1,5^{\circ} \mathrm{C}$, ambos respecto a niveles preindustriales), no se debe per se al soft law. Es la voluntad de los Estados y de la comunidad internacional lo que al fin y al cabo puede hacer la diferencia, porque ya sea soft law o hard law, el diseño y cumplimiento de la norma (la redacción, el lenguaje, lo suave o riguroso, o los mecanismos para hacerla cumplir, haya o no sanción) dependerá en mucho de todos ellos.

Por lo expuesto con antelación, nos parece exagerado el triunfalismo con el que se ha querido caracterizar al AP, al menos desde la perspectiva jurídica. No hay nada de novedoso o espectacular en el hecho de haber adoptado un instrumento hard con mucha normativa soft. Esto se hace con una infinidad de tratados ambientales, y nada parece indicar en nuestros días que este esquema jurídico vaya a cambiar sustancialmente. Era muy ingenuo creer que el AP, con todo y su naturaleza vinculante, hubiera sido normativamente muy riguroso.

\section{BIBLIOGRAFÍA}

AceDO, Alfredo, "Con el Acuerdo de París arderá el planeta", Agencia Latinoamericana de Información (ALAI), 10 de diciembre de 2015, disponible en: wrere.alainet.org/es/articulo/174215.

AnTAL, Edit, Cambio climático: desacuerdo entre Estados Unidos y Europa, México, Plaza y Valdés-UNAM, Centro de Investigaciones sobre América del Norte (CISAN), 2004.

Arellano García, Carlos, Primer curso de derecho internacional público, 7 a. ed., México, Porrúa, 2013.

BAXTeR, R. R., "International Law in «Her Infinite Variety»", The International and Comparative Law Quarterly, vol. 29, núm. 4, 1980. 
Beyerlin, Ulrich y Marauhn, Thilo, International Environmental Law, Oxford, Hart-CH Beck-Nomos, 2011.

BIRNIE, Patricia et al., International Law and the Environment, 3a. ed., Oxford, Oxford University Press, 2009.

BoDAnsky, Daniel, The Art and Craft of International Environmental Law, Cambridge-Londres, Harvard University Press, 2010.

BorRás Pentinat, Susana, Los regímenes internacionales de protección del medio ambiente, Valencia, Tirant lo Blanch, 2011.

Brownlie, Ian, Principles of Public International Lawe, 7a. ed., Oxford, Oxford University Press, 2008.

Cabrera Acevedo, Lucio, El derecho de protección al ambiente, México, UNAM, Instituto de Investigaciones Jurídicas, 1981.

Cárdenas Castañeda, Fabián Augusto, "A Call for Rethinking the Sources of International Law: Soft Law and the Other Side of the Coin", Anuario Mexicano de Derecho Internacional, vol. XIII, 2013.

Casese, Antonio, International Law, 2a. ed., Oxford, Oxford University Press, 2005.

Chinkin, C. M., "The Challenge of Soft Law: Development and Change in International Law", The International and Comparative Law Quarterly, vol. 38, núm. 4, 1989.

De SADELEer, Nicolas, "The Precautionary Principle in International Law", en Kerbrat, Yann y Maljean-Dubois, Sandrine (eds.), The Transformation of International Environmental Law, Oxford-París, A. Pedone \& Hart, 2011.

Diez De Velasco, Manuel, Instituciones de derecho internacional público, 16a. ed., Madrid, Tecnos, 2007.

DiXON, Martin et al., Cases \& Materials on International Lawe, 5a. ed., Oxford, Oxford University Press, 2011.

DuPuY, Pierre-Marie, "Soft Law and the International Law of the Environment", Michigan Fournal of International Law, vol. 12, núm. 420, 1991.

FLORES LIERA, Socorro, "El cambio climático, ¿un reto que rebasa a la comunidad internacional?", en GONZÁLEZ G., Guadalupe et al. (eds.), México y el multilateralismo del siglo XXI. Reflexiones a los 70 años de la ONU, México, Siglo Veintiuno Editores-ITAM-Senado de la República, 2015.

FresnedA, Carlos, "El Acuerdo de París: ¿éxito o fraude?”, El Mundo, 13 de diciembre de 2015, disponible en: wrere.elmundo.es/ciencia/2015/12/ 13/566dc1dfee2704ece3d8b4614.html. 
Esta revista forma parte del acervo de la Biblioteca Jurídica Virtual del Instituto de Investigaciones Jurídicas de la UNAM

GaRvey, James, The Ethics of Climate Change. Right and Wrong in a Warming World, Londres, Continuum, 2008.

Guruswamy, Lakshman, International Environmental Law, 2a. ed., Thomson-West, 2003.

Herdegen, Matthias, Derecho internacional público, trad. de Marcela Anzola, México, Fundación Konrad Adenauer-UNAM, Instituto de Investigaciones Jurídicas, 2005.

IPCG, Cambio climático 2014: Informe de sintesis. Contribución de los Grupos de trabajo I, II y III al Quinto Informe de Evaluación del Grupo Intergubernamental de Expertos sobre el Cambio Climático, Ginebra, IPCG, 2015.

Juste RuIZ, José, Derecho internacional del medio ambiente, Madrid, McGrawHill, 1999.

KIss, Alexandre Ch., Survey of Current Developments in International Environmental Lawe, Morges, IUCN Environmental Policy and Law Paper, núm. 10, 1976.

- y Shelton, Dinah, International Environmental Law, 3a. ed., Nueva York, Trasnational Publishers, 2004.

Klabbers, Jan, "Reflections on Soft International Law in a Privatized World", Lakimies, vol. 104, 2006.

KLEIN, Naomi, Esto lo cambio todo. El capitalismo contra el clima, trad. de Albino Santos Mosquera, Barcelona, Paidós, 2015.

LÓPEZ-BASSOLS, Hermilo, Los nuevos desarrollos del derecho internacional público, 3a. ed., México, Porrúa, 2008.

MaLjEAn-Dubois, Sandrine, "The Making of International Law Challenging Environmental Protection", en KERBRAT, Yann y MALjEANDuBOIS, Sandrine (eds.), The Transformation of International Environmental Law, Oxford-París, A. Pedone \& Hart, 2011.

MÉNDEZ-Silva, Ricardo, "Tratados internacionales", Diccionario jurídico mexicano, 8a. ed., México, Porrúa-UNAM, Instituto de Investigaciones Jurídicas, 1995.

NAVA Escudero, César, "Guía mínima para la enseñanza del derecho internacional ambiental en México", Boletín Mexicano de Derecho Comparado, nueva serie, año XXXVIII, núm. 113, mayo-agosto de 2005.

—, "México ante la COP-16. Entre la simulación climática y la incertidumbre jurídica", Revista del Posgrado en Derecho de la UNAM, núm. 13, julio-diciembre de 2011. 
Palacios Treviño, Jorge, Tratados. Legislación y práctica en México, 4a. ed., México, Secretaría de Relaciones Exteriores-Universidad Iberoamericana, 2007.

Peters, Anne y PAgotto, Isabella, "Soft Law as a New Mode of Governance: A Legal Perspective", NEWGOV: New Modes of Governance, 28 de febrero de 2006, disponible en: http://dx.doi.org/10.2139/ssm.1668531.

Rey Caro, Ernesto J., Temas de derecho internacional ambiental, Córdoba, (Argentina), Marcos Lerner Editora Córdoba, 1998.

RoDILES, Alejandro, "El Acuerdo de París: un empujoncito hacia la justicia climática", Nexos, febrero de 2016.

SANDS, Philippe, Principles of International Environmental Law, 2a. ed., Cambridge, Cambridge University Press, 2003.

SARASíbAR IRIARTE, Miren, El derecho forestal ante el cambio climático: las funciones ambientales de los bosques, Navarra, Thomson-Aranzadi, 2007.

SeAra VÁzQUez, Modesto, Derecho internacional público, 24a. ed., México, Porrúa, 2012.

Sepúlveda, César, Derecho internacional, 20a. ed., México, Porrúa, 2000.

SHAw, Malcolm N., International Law, 7a. ed., Cambridge, Cambridge University Press, 2014.

Shelton, Dinah, "Comments on the Normative Challenge of Environmental «Soft Law»", en Kerbrat, Yann y MaLjean-Dubois, Sandrine (eds.), The Transformation of International Environmental Law, OxfordParís, A. Pedone \& Hart, 2011.

Solón, Pablo, "COP21: A New Disguise for an Old Agreement", noviembre de 2015, disponible en: https://pablosolon.wordpress.com.

SzÉKELY, Alberto, "El medio ambiente: derecho internacional", Revista de la Facultad de Derecho de México, México, t. XXVI, núms. 103-104, juliodiciembre de 1976.

y Ponce-NAVA, Diana, "La Declaración de Río y el derecho internacional ambiental", en GLENDER, Alberto y LiCHTINGER, Víctor, La diplomacia ambiental. México y la Conferencia de las Naciones Unidas sobre Medio Ambiente y Desarrollo, México, Secretaría de Relaciones ExterioresFondo de Cultura Económica, 1994.

TORO Huerta, Mauricio Iván del, "El fenómeno del soft law y las nuevas perspectivas del derecho internacional", Anuario Mexicano de Derecho Internacional, vol. VI, 2006. 
Esta revista forma parte del acervo de la Biblioteca Jurídica Virtual del Instituto de Investigaciones Jurídicas de la UNAM

United Nations Framework Convention on Climate Change, Synthesis Report on the Aggregate Effect of INDCs, UNFCG, 2015, disponible en: unfccc.int/focus/indc_portal/items/9240.php.

United Nations Organization, "Definitions of Key Terms Used in the UN Treaty Collection", United Nations, Treaty Collection, disponible en: https://treaties.un.org/Pages/overview.aspx?path=overview/definition/page1_ en.xml.

Vallarta Marrón, José Luis, Derecho internacional público, 2a. ed., México, Porrúa, 2014.

VAN Lierop, Robert F. y SzÉKely, Alberto, "Compliance with Environmental Treaties: The Empirical Evidence a Commentary on the Softening of International Environmental Law", Proceedings of the Annual Meeting, vol. 91, 1997.

WeIL, Prosper, "Towards Relative Normativity in International Law?", The American Fournal of International Law, vol. 77, núm. 3, 1983.

Villoro Toranzo, Miguel, Introducción al estudio del derecho, 21a. ed., México, Porrúa, 2012.

VIÑUALES, Jorge, "The Paris Climate Agreement: An Initial Examination (Part II of III)", EfIL: Talk! Blog of the European Fournal of International Law, 8 de febrero de 2016, disponible en: wrere.ejiltalk,org/the-pari-climateagreement-an-initial-examination-part-ii-of-iii/.

-, "The Paris Climate Agreement: An Initial Examination (Part III of III)", EfIL: Talk! Blog of the European Fournal of International Law, $8 \mathrm{de}$ febrero de 2016, disponible en: wrere.ejiltalk,org/the-pari-climate-agreementan-initial-examination-part-iii-of-iii/.

Williamson, Richard L. Jr., "Hard Law, Soft Law, and Non-Law in Multilateral Arms Control: Some Compliance Hypotheses", Chicago fournal of International Law, vol. 4, núm. 1, 2003. 